\title{
Classical Fluids of Negative Heat Capacity
}

\author{
P. T. Landsberg
}

Faculty of Mathematical Studies

University of Scuthampton

DOE/ER/40272--166

Southampton SO9 $5 \mathrm{NH}, \mathrm{UK}$

and

R. P. Woodard

Department of Physics

University of Florida

Gainesville, FL 32611, USA

ABSTRACT: It is show that new parameters $X$ can be defined such that the heat capacity $C_{X} \equiv T\left(\frac{\partial S}{\partial T}\right) X$ is negative, even when the canonical ensemble (i.e. at fixed $T=\left(\frac{\partial l}{\partial S}\right)_{Y^{\prime}}$ and $\left.I^{\prime} \neq X\right)$ is stable. As examples we treat black body radiation and general gas systems with nonsingular $\kappa_{T}$. For the case of a simple ideal gas we even exhibit an apparatus which enforces a constraint $X(p, V)=$ const. that makes $C, X<0$. Since it is possible to invent constraints for which canonically stable systems have negative heat capacity we speculate that it may aiso be possible to infer the statistical mechanics of canonically unitable systems - for which even the traditionad heat capacities are negative - by imposing constraints that stabilize the associated, noncanonical ensembles. 
The idea of negative heat capacities for black holes [1], and for interacting classical systems like stars [2], is by now well known. The physical realization of many familiar thermodynamic constructions is problematic in both cases because of the important role played by gravitation [3]. In view of the long range and universal character of this interaction it is very doubtful that a thermal reservoir which is large enough to enforce a sharply defined temperature would not also completely dominate the gravitational dynamics of any small system to which it was coupled. This is an important practical problen in the thermodynamics of gravitation but it should not be allowed to obscure the fundamental problem that a system whose heat capacity is negative cannot reach thermal equilibrium even with an idealized reservoir.

The physical argument is of course that a system with negative heat capacity warms up by losing heat and cools down by gaining it. If heat flows from hot to cold then any temperature difference with respect to the reservoir will engender heat flows which increase the difference. The mathematical argument proceeds by contradiction. Let $\hat{H}$ represent the system's Haniltonian and let $Y$ stand for the usual extensive parameters in addition to the energy. (This discussion can be generalized to include the case where any of the extensive parameters $Y_{i}$ is replaced by its intensive conjugate, $P_{i} \equiv\left(\frac{\partial U}{\partial Y_{i}}\right)$. See, for example, [4].) If the system is in equilibrium with a reservoir at fixed $Y$ and fixed temperature $T=\left(\frac{\partial U}{\partial S}\right)_{Y}$ then elementary statistical mechanics gives the canonical partition function:

$$
Z(T, Y) \equiv \operatorname{Tr}\left(e^{-\beta \widehat{H}}\right)_{Y}
$$

where $\beta \equiv \frac{1}{k_{B} T}$ and the trace extends over all states of fixed $Y$. Taking the logarithm gives $-\beta$ times the Helmholtz free energy, $F(T, Y)$. Using the standard formulae for the entropy anc heat capacity, $S=-\left(\frac{\partial F}{\partial T}\right)_{Y}$ and $C_{Y} \equiv T\left(\frac{\partial S}{\partial T}\right)_{Y}$, we find that the usual heat capacity is proportional to the variance of the energy:

$$
C_{Y}=k_{B} \beta^{2}\left(\frac{\partial^{2} \ln (Z)}{\partial \beta^{2}}\right)_{Y}=k_{B} \beta^{2}\left\langle(\hat{H}-\langle\hat{H}\rangle)^{2}\right\rangle
$$


This is a manifestly positive quantity, so the assumption of thermal equilibrium must be erroneous whenever $C_{Y}$ is negative.

In view of the preceding arguments it is usually assumed that systems with negative heat capacity can be treated statistically only in the microcanonical ensemble. We have just seen the problem when it is the usual heat capacity, $C_{Y}$, which is negative, but oue might ask how the matter stands for a more general heat capacity, $C_{X} \equiv T\left(\frac{\partial S}{\partial T}\right)_{X}$, where $X$ is not restricted to be the extensive parameter(s) of the canonical ensemble. The answer is that quantities $X$ can always be defined such that $C_{X}$ is less than zero, even when $C_{Y}$ is positive and the canonical ensemble is completely stable [5], pp. 42, 45. Examples of functions $X$ will be given below. The physical picture is of a system coupled to a reversible work source that adds or withdraws energy so as to keep some quantity $X$ fixed. The system will have $C_{X}<0$ if the constraint $X=$ const. is chosen so that any heat flow from the reservoir is overbalanced by the work drawn off by the reversible work source.

We will prove the existence of such constraints generally in the context of normal thermodynamics where the natural extensive parameters are internal energy and volume. This context entirely avoids systems based upon the sort of long range and universal forces that would mediate strong interactions with the reservoir. We first give the general solution under the assumption that the isothermal compressibility $\left(\kappa_{T} \equiv-\frac{1}{v}\left(\frac{\partial v}{\partial p}\right)_{T}\right)$ is nonsingular, then we give a detailed mechanical realization of such a constraint for the simple ideal gas. To illustrate that singular ${ }_{i} T$ need not pose a problem we show how negative heat capacities can be defined also for black body radiation.

Although our parameters $X$ can be extensive, for canonically stable systems they necessarily exhibit the following properties in contrast to the usual parameter $Y=V$ :

$$
\begin{gathered}
C_{X}=T\left(\frac{\partial S}{\partial T}\right)_{X} \neq\left(\frac{\partial U}{\partial T}\right)_{X} \\
T=\left(\frac{\partial U}{\partial S}\right)_{Y} \neq\left(\frac{\partial U}{\partial S}\right)_{X} \equiv T^{\prime}
\end{gathered}
$$


The final inequality means that the statistical ensemble at constant $T$ and $X$ is not weighted by the Boltzmann factor of (1a), as is the ensemble at constant $Y$. In fact the ensemble at constant $X$ fails even to exist; the instability of any such system can be seen from the previously cited physical argument. Our results are nonetheless derived using the fundamental relation obtained from the canonical ensemble at fixed $T$ and $V$. This suggests that by choosing different extensive parameters one might be able to explore the statistical mechanics of a system with negative $C_{V}$ - in which case it would be the noncanonical ensemble at fixed $T^{\prime}$ and $X$ which would exist rather than the canonical ensemble at fixed $T$ and $V$ - without recourse to the microcanonical ensemble.

Let $X(p, V)$ be some function of pressure and volurne. Then one can write for the incremental heat input into the system [5], pp. 11, 22:

$$
\begin{aligned}
T d S & =C_{V} d T+\ell_{V} d V=\left[C_{V}+\ell_{V}\left(\frac{\partial V}{\partial T}\right)_{X}\right] d T+\ell_{V}\left(\frac{\partial V}{\partial X}\right)_{T} d X \\
& =C_{p} d T+\ell_{p} d p=\left[C_{p}+\ell_{p}\left(\frac{\partial p}{\partial T}\right)_{X}\right] d T+\ell_{p}\left(\frac{\partial p}{\partial X}\right)_{T} d X \\
& =C_{X} d T+\ell_{X} d X
\end{aligned}
$$

where $\ell_{X}$ is the appropriate "latent heat". The two expressions which result for $\ell_{X}$ are:

$$
\begin{aligned}
& \ell_{X}=\ell_{V}\left(\frac{\partial V}{\partial X}\right)_{T}=T\left(\frac{\partial p}{\partial T}\right)_{V}\left(\frac{\partial V}{\partial X}\right)_{T} \\
& \ell_{X}=\ell_{p}\left(\frac{\partial p}{\partial X}\right)_{T}=-T\left(\frac{\partial V}{\partial T}\right)_{p}\left(\frac{\partial p}{\partial X}\right)_{T}
\end{aligned}
$$

Their consistency is a simple consequence of the reciprocity theorem:

$$
\left(\frac{\partial p}{\partial T}\right)_{V}\left(\frac{\partial T}{\partial V}\right)_{p}\left(\frac{\partial V}{\partial p}\right)_{T}=-1
$$

Note that $\ell_{X} d X$ is not generally the work done on the system, although $\ell_{V} d V=p d V$ for the special case of the simple, ideal gas. From (3) we can infer two expressions for $C_{X}$ :

$$
\begin{aligned}
C_{X} & =C_{V}+\ell_{V}\left(\frac{\partial V}{\partial T}\right)_{X} \\
& =C_{p}+\ell_{p}\left(\frac{\partial p}{\partial T}\right)_{X}
\end{aligned}
$$


Subtracting $C_{V}$ from (6a) and taking the ratio with $(6 b)-(6 a)$ gives:

$$
\frac{C_{X}-C_{V}}{C_{p}-C_{V}}=\frac{1}{1-\theta} \quad \text {, i.e. } \quad \frac{C_{X}}{C_{V}}=-\frac{\gamma-\theta}{\theta-1}
$$

where:

$$
\theta \equiv \frac{\ell_{p}}{\ell_{V}}\left(\frac{\partial p}{\partial V}\right)_{X}=\left(\frac{\partial V}{\partial p}\right)_{T}\left(\frac{\partial p}{\partial V}\right)_{X}
$$

Note that $\theta$ depends only upon the definition of $X$ and an equation of state relating $V, p$ and $T$.

The generalized heat capacity $C_{X}$ was introduced long ago [5], pp. 42, 45. It has also been studied recently in the context of enforced adiabats, i.e. processes in which differential heat flow can occur along a path provided the initial and final entropies are equal [6]. If $X$ is a function of $p$ alone then $\theta=0$ and $C_{X}=\gamma C_{V}=C_{p}$. Also one sees from (8) that if $X$ is a function of $V$ only then $\theta \rightarrow \infty$, and Fig. 1 confirms $C_{X} \rightarrow C_{V}$. Thus $C_{X}$ covers a range of heat capacities which include $C_{V}($ at $\theta \rightarrow \infty)$ and $C_{p}($ at $\theta=0)$.

To show that negative values are possible note that $C_{X}<0$ if and only if $1<\theta<\gamma$. Now let $\theta_{0}$ be a constant in this range and consider the relation $\theta=\theta_{0}$. Identification of the isothermal compressibility $\kappa_{T}$ and simple applications of the reciprocity and inverse theorems give:

$$
\kappa_{T}(p, V) V\left(\frac{\partial X}{\partial V}\right)_{p}=\theta_{0}\left(\frac{\partial X}{\partial p}\right)_{V}
$$

This is a linear partial differential equation with possibly nonconstant but certainly nonzero coefficients. We can of course freely specify $\mathrm{X}\left(p_{0}, V\right)$ for some fixed pressure $p_{0}$ and the general solution follows by exponentiation:

$$
X(p, V)=\mathrm{P}\left(\exp \left[\frac{1}{\theta_{0}} \int_{p_{0}}^{p} d z \kappa T(z, V) V \frac{\partial}{\partial V}\right]\right) \mathrm{X}\left(p_{0}, V\right)
$$

The symbol "P" stands for "pat'h-ordering." It means that the various noncommuting factors of $\kappa_{T}(z, V) V \frac{\partial}{\partial V}$ are to be ordered according to the $z$ integration with those nearer 
$z=p$ to the left of those nearer $z=p_{0}$. Generally if $\mathcal{O}(z)$ is an operator or matrix-valued function of $z$ then one makes the following definition:

$$
\mathrm{P}\left(\exp \left[\int_{a}^{b} d z \mathcal{O}(z)\right]\right) \equiv \sum_{n=0}^{\infty} \int_{a}^{b} d z_{1} \int_{z_{1}}^{b} d z_{2} \ldots \int_{z_{n-1}}^{b} d z_{n} \mathcal{O}\left(z_{n}\right) \mathcal{O}\left(z_{n-1}\right) \ldots \mathcal{O}\left(z_{1}\right)
$$

To make the preceding discussion more concrete suppose that the entropy has the form appropriate to a simple ideal gas:

$$
S(U, V, N)=k_{B} N \ln \left[\left(a \frac{U}{N}\right)^{\frac{1}{\gamma-1}} \frac{V}{N}\right]
$$

where $N$ is the number of particles and $a$ is related to the chemical constant $i$. In fact, if $\gamma=\frac{5}{3}$ then $a=\frac{2}{3}\left(k_{B}\right)^{-\frac{5}{3}} \exp \left(\frac{2}{3} i\right)$. A simple exercise reveals that $\kappa_{T}=\frac{1}{p}$. Substituting this into expression (10) gives:

$$
X(p, V)=X\left(p_{0}, V\left[\frac{p}{p_{0}}\right]^{\frac{1}{\theta_{0}}}\right)
$$

In other words, $X(p, V)$ can be any function of $V p^{1 / \theta_{0}}$. Since keeping $X$ fixed is the same as keeping $V p^{1 / \theta_{0}}$ fixed we can make $X$ extensive by taking $X(p, V)=V p^{1 / \theta_{0}}$. In fact, the negative nature of $C_{X}$ zan be obtained directly and very simply from this expression for $X(p, V)$.

A primitive apparatus is depicted in Fig. 2. A thermal reservoir of temperature $T_{\text {res }}$ surrounds a large, diathermal cylinder of cross-sectional area $A$. The cylinder contains a movable piston which is attached to a spring whose force constant $k$ can be stiffened or loosened by winding or unwinding the coils of the spring. The piston seals a fixed quantity of the gas into one end of the cylinder while the other end is vacuum. Transducers measure the length $L$ of the gas-filled section and the pressure, $p=k: L / A$. These transducers control the winding mechanism which adjusts the spring's force constant according to the rule:

$$
k(L)=\frac{k_{0}}{L^{\theta_{0}+1}}
$$


where $k_{0}$ is a constant. It is easy to see that this serves to maintain the constraint $V p^{1 / \theta_{0}}=$ constant.

To determine the state of the system we allow energy to flow so as to maximize the total entropy of the three components: the gas, the spring and the reservoir. The energy of the spring is simple to compute from Hooke's Law:

$$
U_{\text {spring }}=\int_{L_{0}}^{L} d x k(x) x=\text { const. }-\frac{1}{\theta_{0}-1} p V
$$

Since $U_{\text {gas }}=\frac{1}{\gamma-1} p V$ it follows that $U_{\text {spring }}=$ const. $-\left(\frac{\gamma-1}{\theta_{0}-1}\right) U_{\text {gas. }}$. Now suppose that the internal energy of the gas increases by an amount $\Delta U_{\text {gas. }}$ The preceding relation and energy conservation imply that the corresponding increases in the spring and reservoir energies are:

$$
\begin{gathered}
\Delta U_{\text {spring }}=-\left(\frac{\gamma-1}{\theta_{0}-1}\right) \Delta U_{\text {gas }} \\
\Delta U_{\text {res }}=\left(\frac{\gamma-\theta_{0}}{\theta_{0}-1}\right) \Delta U_{\text {gas }}
\end{gathered}
$$

To compute the change in entropy note that with $X=V p^{1 / \theta_{0}}$ we can express the volume in terms of $X$ and $U$ :

$$
V=X^{\frac{\theta_{0}}{\theta_{0}-1}}\left[(\gamma-1) U_{\mathrm{gas}}\right]^{-\frac{1}{\theta_{0}-1}}
$$

Substitution into (11) gives the entropy of the gas as:

$$
S_{\text {gas }}=-\frac{k_{B} N}{\gamma-1}\left(\frac{\gamma-\theta_{0}}{\theta_{0}-1}\right) \ln \left(U_{\text {gas }}\right)+f(X, N)
$$

where $f(X, N)$ is a simple function whose precise form is arrelevant to our discussion. If $T_{\text {gas }}$ is the gas temperature before the addition of the increment $\Delta U_{\text {gas }}^{*}$ then the gas entropy increases by:

$$
\Delta S_{\text {gas }}=-\frac{1}{T_{\text {gas }}}\left(\frac{\gamma-\theta_{0}}{\theta_{0}-1}\right) U_{\text {gas }} \ln \left(1+\frac{\Delta U_{\text {gas }}}{U_{\text {gas }}}\right)
$$

The reservoir is a reversible heat source at constant temperature so its entropy increases by:

$$
\Delta S_{\mathrm{res}}=\frac{1}{T_{\mathrm{res}}}\left(\frac{\gamma-\theta_{0}}{\theta_{0}-1}\right) \Delta t_{\mathrm{gas}}
$$


The spring and its winding mechanism are assumed to constitute a reversible work source so their entropies are unchanged. For $1<\theta_{0}<\gamma$ we see that the total entropy can be made to increase without bound by having $\Delta U_{\text {gas }}$ approach $-U_{\text {gas. }}$. Hence heat flows from the reservoir even as the gas expands and cools down; the excess energy goes into unwinding the spring. This is precisely the sort of instability that one expects from a system with negative heat capacity.

So much for our mechanical contraption. Provided that the isothermal compressibility, $\kappa_{T}$, is nonsingular expression (10) gives the general solution for a constraint $X(p, V)$ such that $C_{X}=-\left(\frac{\gamma-\theta_{0}}{\theta_{0}-1}\right) C_{V}$. In fact negative heat capacities can be defined even for systems where both $\kappa_{T}$ and $C_{p}=\gamma C_{V}$ are singular. To illustrate this fact we consider black body radiation. A fundamental relation for this system is:

$$
S(U, V)=\frac{4}{3} a^{\frac{1}{4}} V^{\frac{1}{4}} U^{\frac{3}{4}}
$$

where $a$ is the Stefan-Roltzm ann constant. Some differentiations and rearrangements suffice to give the standard results:

$$
\begin{aligned}
& U=a V T^{4} \\
& p=\frac{1}{3} a T^{4}
\end{aligned}
$$

The latter implies that $\kappa_{T}$ and $C_{p}$ are infinite for this system.

To obtain a negative heat capacity let us „gain consider extensive constraints of the form:

$$
X(p, V)=V p^{\frac{1}{\not}}
$$

By using (19b) to solve for $T(V, X)$ and then substituting into (19a) we obtain the following expressions for $V^{\prime}$ :

$$
V=\left(\frac{1}{3} U\right)^{\frac{1}{1-\theta}} X^{\frac{-\theta}{1-\theta}}=\left(\frac{u}{3} T^{4}\right)^{-\frac{1}{\theta}} X
$$


These relations allow us to write the entropy in terms of either $U$ and $X$ or $T$ and $X$ :

$$
\frac{1}{4} S=3^{\left(\frac{-5+4 \theta}{4-4 \theta}\right)} a^{\frac{1}{4}} X^{\left(\frac{-\theta}{4-4 \theta}\right)} U^{\left(\frac{4-3 \theta}{4-4 \theta}\right)}=\left(\frac{a}{3}\right)^{\frac{\theta-1}{\theta}} X T^{\left(\frac{3 \theta-4}{\theta}\right)}
$$

To see that $\theta$ can be chosen to make the system at fixed $U$ and $X$ unstable we merely differentiate the first of these expressions twice:

$$
\left(\frac{\partial^{2} S}{\partial U^{3}}\right)_{X}=\frac{4 \theta-3 \theta^{2}}{(4-4 \theta)^{2}} \frac{S}{U^{2}}
$$

Concavity obviously fails for $0<\theta<\frac{4}{3}$. That this range of values also corresponds to a nef ative heat capacity follows from differentiation of the second incarnation of the entropy in (22):

$$
C_{X} \equiv T\left(\frac{\partial S}{\partial T}\right)_{X}=\left(1-\frac{4}{3 \theta}\right) C_{V}
$$

Of course the limit $\theta \longrightarrow \infty$ just recovers $C_{V}$.

We stress that all of the preceding analysis was carried out using conventional thermodynamics and fundamental relations - (11) and (18) - which follow from the canonical ensemble. We are therefore led to suggest that the whole process might be profitably inverted when it is desired to study a system for which the conventional heat capacity, $C_{Y}$, is negative. That is, it might be possible define a parameter $X(U, Y)$ such that a noncanonical ensermble is "stable," whose partition function is:

$$
Z^{\prime}\left(Y^{\prime}, X\right)=\operatorname{Tr}\left[\exp \left(-\frac{\hat{H}}{k_{B} T^{\prime}}\right)\right]_{X}
$$

where $T^{\prime}$ is given by equation (2b). By "stable" we mean that the function $F^{\prime}\left(T^{\prime}, X\right) \equiv$ $-k_{B} T^{\prime} \ln \left[Z\left(T^{\prime}, X\right)\right]$ is convex with respect to $T^{\prime}$ and concave with respect to $X$. One would then eliminate $T^{\prime}$ by inverting the relation:

$$
S=-\left(\frac{\partial F^{\prime}}{\partial T^{\prime}}\right)_{X}
$$

Substituting $T^{\prime}(S, X)$ into the internal energy:

$$
U=-k_{B} T^{\prime 2}\left(\frac{\partial \ln \left(Z^{\prime}\right)}{\partial T^{\prime}}\right)_{X}
$$


and inverting to solve for $S=S(U, X(U, Y))$ would give the desired fundamental relation in the entropic representation. It might be very much simpler to infer the statistical mechanics of an unstable system in this way than by going through the microcanonical ensemble.

\section{ACKNOWLEDGEMENT}

This work was partially supported by DOE contract DE-FG05-86-ER40272.

\section{DISCLAIMER}

This report was prepared as an account of work sponsored by an agency of the United States Government. Neither the United States Government nor any agency thereof, nor any of their employees, makes any warranty, express or implied, or assumes any legal liability or responsibility for the accuracy, completeness, or usefulness of any information, apparatus, product, or process disclosed, or represents that its use would not infringe privately owned rights. Reference herein to any specific commercial product, process, or service by trade name, trademark, manifacturer, or otherwise does not necessarily constitute or imply its endorsement, recommendation, of favoring by the United States Government or any agency thereof. The views and opinions of authors expressed herein do not necessarily state or reflect those of the Unitod States Government or any agency thereof. 


\section{REFERENCES}

[1] P. C. W. Davies, Rep. Prog. Phys. 41 (1978) 1314;

R. M. Wald, General Relativity (Chicago University Press, Chicago, 1984);

D. Pavón and P. T. Landsberg, Gen. Rel. and Grav. 20 (1988) 457.

[2] D. Lynden-Bell and R. M. Lynden-Bell, Mon. Not. Roy. Astro. Soc. 181 (1977) 405 .

P. Hertel and W. Thirring, Annals of Physics 63 (1971) 520.

[3] P. T. Landsberg, J. Stat. Phys. 35 (1984) 159.

D. C. Wright, Phys. Rev. D21 (1980) 884.

[4] F. Schlögl, Z. Phys. 267 (1974) 77.

[5] P. T. Landsberg, Thermodynamics and Quantum Statistical Illustrations (Interscience, New York, 1961).

[6] V. J. Menon and D. C. Agrawal, Phys. Lett. 139A (1989) 130.

\section{FIGURE CAPTIONS}

1. Plot of the generalized heat capacity $C_{X}$ (in units of $C_{V}$ ) versus the quantity $\theta$ defined in equation (8). Note that each value of $\theta$ corresponds to a different choice of the quantity $X(p, V)$ which is held fixed.

2. Sketch of a system which realizes negative heat capacity using a simple ideal gas. 


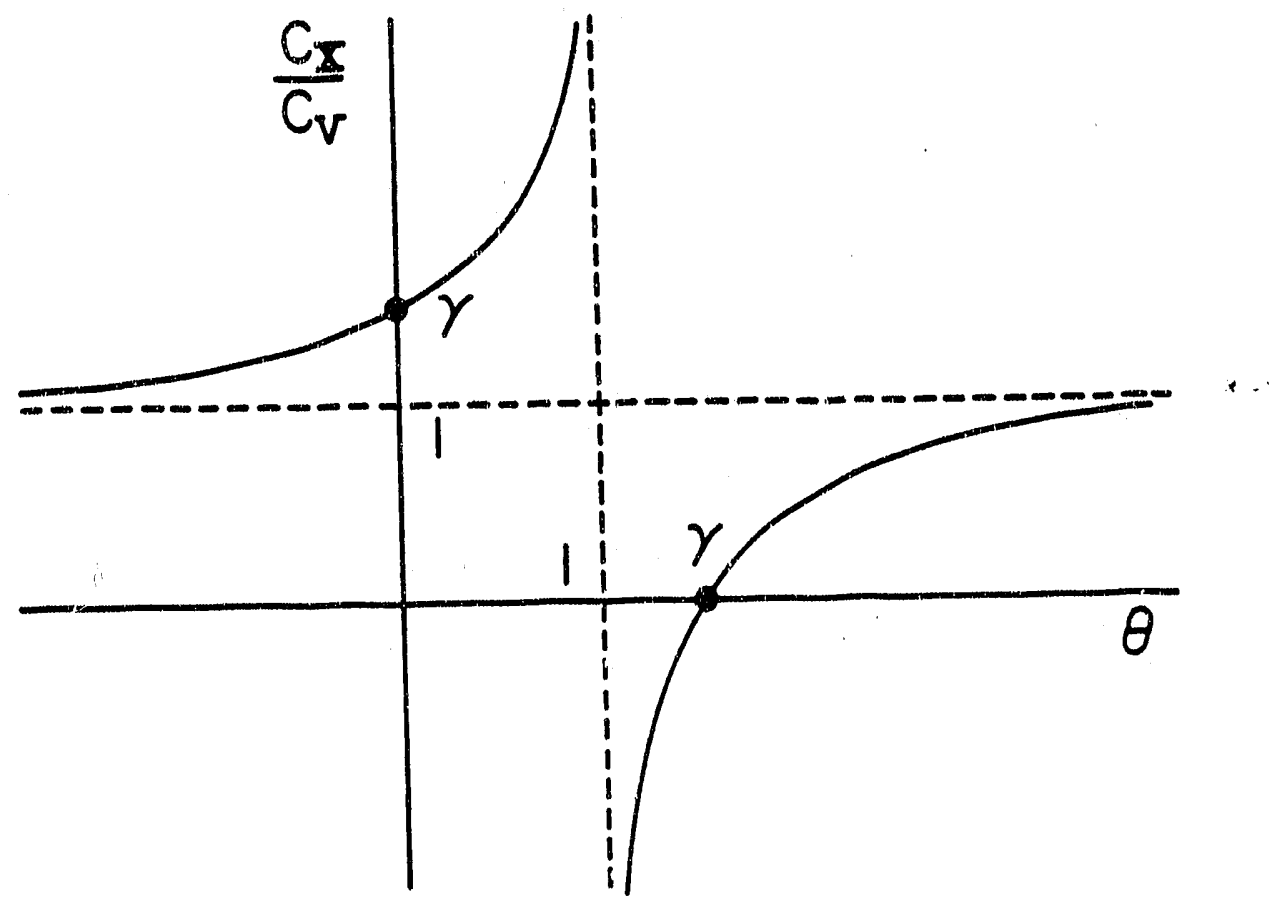

Landsberg and Woodard

"Classical Fluids of ..."

Figure 1 


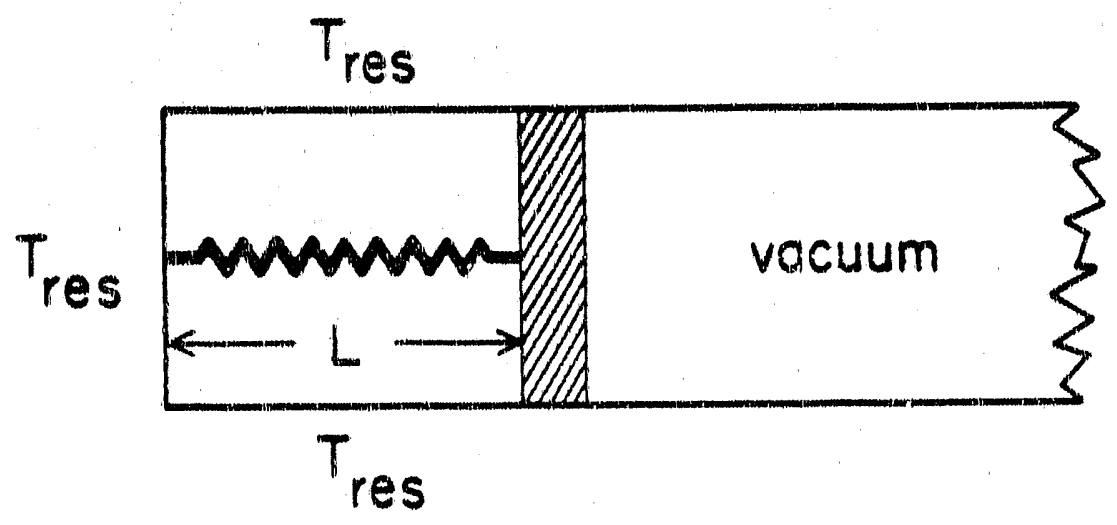

Landsberg and Woodard

"Classical Fluids of ..."

Figure 2 

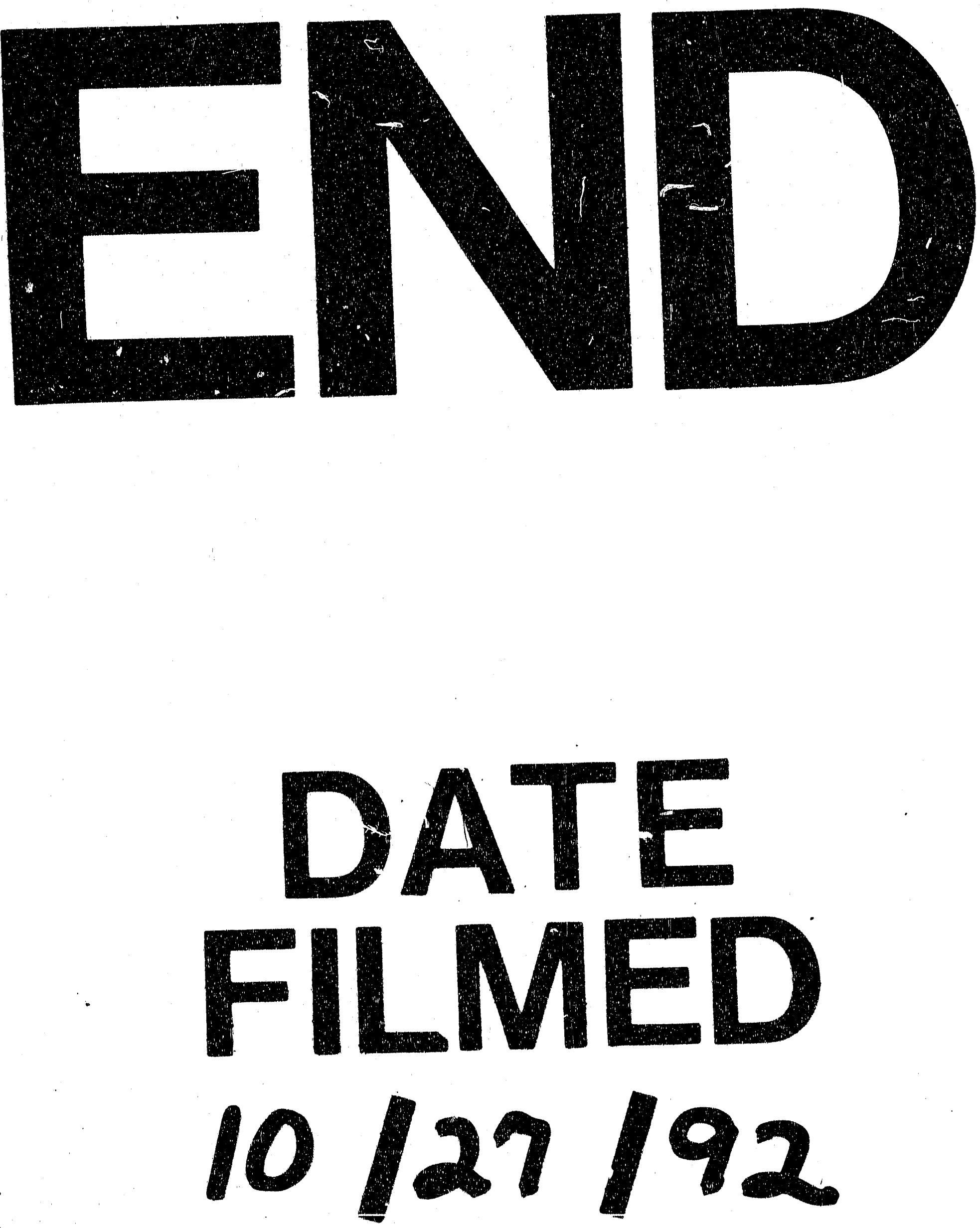
